\title{
Immune Cell
}

National Cancer Institute

\section{Source}

National Cancer Institute. Immune Cell. NCI Thesaurus. Code C132890.

A cell in the immune system that is involved in host defense. This category may include lymphocytes, monocytes, macrophages, neutrophils, eosinophils, basophils, mast cells, and thrombocytes. Precursor cells in these lineages may also be included. 